\title{
Fuzzy contra S-irresolute continuous mappings in Ŝostak's fuzzy topological spaces
}

\author{
B. Vijayalakshmi ${ }^{1 *}$, P. Dhanasekaran ${ }^{2}$, M. Angayarkanni ${ }^{3}$, and A. Vadivel ${ }^{4}$
}

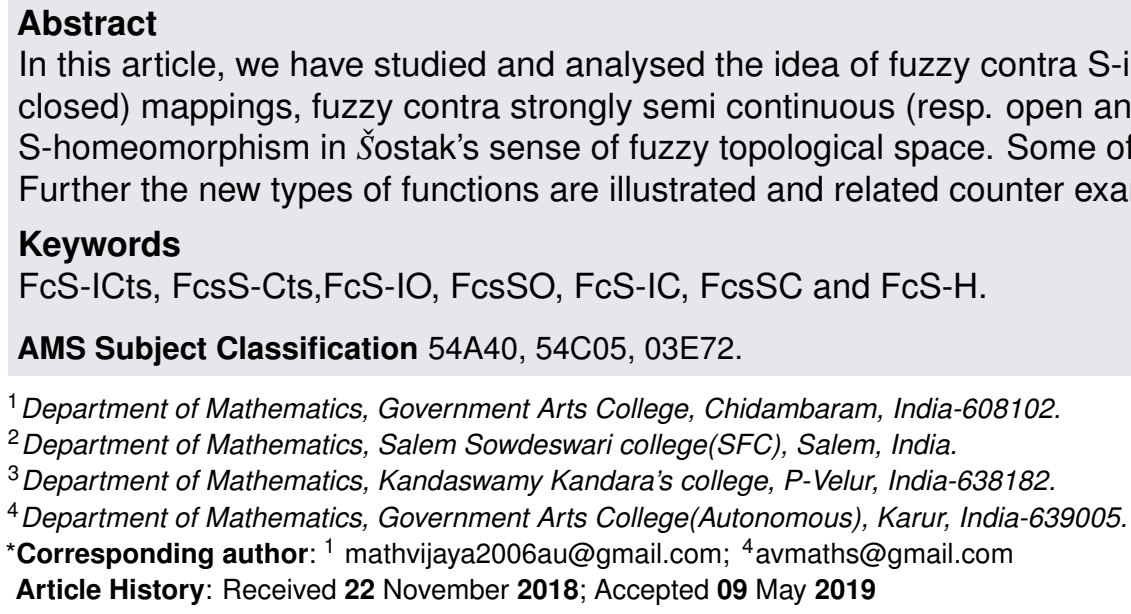

\section{Contents}

1 Introduction and Preliminaries. .228 Fuzzy Contra S-irresolute Continuous Mapping...229

References .233 233

\section{Introduction and Preliminaries}

After the introduction of fuzzy sets by Zadeh [18], Chang[1] was the one who initiated the idea of a fuzzy topology in a set $X$, in which a collection, $T$, of open sets of fuzzy subsets of $X$. He also defined new type of fuzzy topology. In [3, 4], the authors defined the idea of gradation of openness of fuzzy subsets of $X$. They also used the concept of fuzzy topology in the sense of Chang. In 1980 in [5],the basic idea of topology is itself fuzzy. As in 1985 by the customized work of Kubiak [8] and Šstak [13] in Lattice $L=I$ was initiated in 1995 by [6] and further by [9] in 1997, in that topology was used to explain the two membership latices namely $L^{X}$ to $M$, where $L$ and $M$ are appropriate lattices. With all the basic ideas of topologies the basic lattices are fixed in $[8,13]$ and another significant generalizations of lattices $L$ varies from space to space [7] is also represented. Using the developments of [5-
9, 12-16], the notion of a fuzzy topology as a fuzzy subset tof powerset is independently consider with the base work of [5, 8, 13]. At present authors Chattopadhyay [3] et.al., Ramadan [11] and Ying [17] are also worked in this concept.

In this article, we defined a new concept of the fuzzy contra s-irresolute continuous (resp. open and closed) mapping, fuzzy contra strongly semi continuous (resp. open and closed) mapping and fuzzy contra s-homeomorphism as the base definition of Šostak and established defined some characteristic properties.

$X, Y$ etc., denotes the non empty sets, $I=[0,1]$ and $I_{0}=(0,1]$. In 1992 Ramadan [11] given the definition of smooth topological spaces (in short, sts) and the operators $C_{\tau}: I^{X} \times I_{0} \rightarrow I^{X}$ as $C_{\tau}(\lambda, r)=\bigwedge\left\{\mu \in I^{X}: \lambda \leq \mu, \tau(\overline{1}-\right.$ $\mu) \geq r\}$. and $I_{\tau}: I^{X} \times I_{0} \rightarrow I^{X}$ as $I_{\tau}(\lambda, r)=\bigvee\left\{\mu \in I^{X}: \lambda \geq\right.$ $\mu, \tau(\mu) \geq r\}$. Kim et. al.[10] discussed in a sts $(X, \tau)$, $\forall r \in I_{0} . \quad \lambda$ is called $r$-fuzzy strongly semiopen (resp. $r$ fuzzy semiopen $\& r$-fuzzy preopen) ( $r$-fsso (resp. $r$-fso $\& r$ fpo) for short) iff $\lambda \leq I_{\tau}\left(C_{\tau}\left(I_{\tau}(\lambda, r), r\right), r\right)$ (resp. $\lambda \leq$ $\left.C_{\tau}\left(I_{\tau}(\lambda, r), r\right) \& \lambda \leq I_{\tau}\left(C_{\tau}(\lambda, r), r\right)\right)$. The respective compliment sets are closed set. The operators $r$-fuzzy strongly semi-interior (resp. $r$-fuzzy strongly semi-closure) of $\lambda$, denoted by $S S I_{\tau}(\lambda, r)$ (resp. $S S C_{\tau}(\lambda, r)$ ) as $S S I_{\tau}(\lambda, r)=\bigvee\{\mu \in$ $I^{X}: \mu \leq \lambda, \mu$ is $r$-fsso $\}$ (resp. $\operatorname{SSC}_{\tau}(\lambda, r)=\bigwedge\left\{\mu \in I^{X}: \mu \geq\right.$ $\lambda, \mu$ is $r$-fssc $\}) . \quad f:(X, \tau) \rightarrow(Y, \eta)$ is fuzzy continuous (FCts for short) iff $\eta(\mu) \leq \tau\left(f^{-1}(\mu)\right)$ for each $\mu \in I^{Y}$. 
$f:(X, \tau) \rightarrow(Y, \eta)$ is fuzzy semi continuous, (resp. fuzzy irresolute and fuzzy S-irresolute continuous) (FSC (resp. FI and FS-IC) for short) iff $f^{-1}(\mu)$ is $r$-fso (resp. $r$-fso and $r$ fsso) set of $X$ for each $\eta(\mu) \geq r$, (resp. $\mu$ is $r$-fso and $r$-fsso )set of $Y r \in I_{0} . f:(X, \tau) \rightarrow(Y, \eta)$ is fuzzy open(resp. fuzzy closed ) (FO (resp. FC) for short) iff $\tau(\lambda) \leq \eta(f(\lambda))$ (resp. $\tau(\overline{1}-\lambda) \leq \eta(\overline{1}-f(\lambda)))$ for each $\lambda \in I^{X} . f:(X, \tau) \rightarrow(Y, \eta)$ is fuzzy S-irresolute open (resp. fuzzy strongly semi-open and fuzzy strongly semi-closed) (FS-IO (resp. FsSO and FsSCC) for short) iff $f(\mu)$ is $r$-fsso (resp. $r$-fsso and $r$-fssc) set of $Y$ for each $r$-fsso $\left(\operatorname{resp} . \tau(\mu) \geq r \& \operatorname{tau}(\overline{1}-\mu) \geq r\right.$, ) set $r \in I_{0}$. $f:(X, \tau) \rightarrow(Y, \eta)$ is fuzzy contra continuous (FcCts, for short) if for each $\mu \in I^{Y}$ we have $\tau\left(\overline{1}-f^{-1}(\mu)\right) \geq \eta(\mu)$. $f:(X, \tau) \rightarrow(Y, \eta)$ is fuzzy contra open(resp. fuzzy contra closed) ( $\mathrm{FcO}$ (resp. $\mathrm{FcC}$ ) for short) if for each $\lambda \in I^{X}$ we have $\eta(\overline{1}-f(\lambda)) \geq \tau(\lambda)$ (resp. $\eta(f(\lambda)) \geq \tau(\overline{1}-\lambda)$ ). The pair $(X, \tau)$ is called a smooth topological space (or) Šostak Fuzzy topological space (or) fuzzy topological spaces (in short, sts, sfts, fts).

\section{Fuzzy Contra S-irresolute Continuous Mapping}

Definition 2.1. Let $(X, \tau)$ and $(Y, \eta)$ be fts's and let $f: X \rightarrow$ $Y$ be a mapping. Then $f$ is called

(1) fuzzy contra S-irresolute continuous (resp. fuzzy contra strongly semi-continuous) (FcS-ICts (resp. FcsSCts) for short) iff $f^{-1}(\mu)$ is $r$-fssc set of $X$ for each $r$-fsso set $\mu \in I^{Y}$ (resp. $\left.\eta(\mu) \geq r\right), r \in I_{0}$.

(2) fuzzy contra S-irresolute open (resp. fuzzy contra strongly semi-open fuzzy contra S-irresolute closed and fuzzy contra strongly semi-closed) (FcS-IO (resp. FcsSO FcS-IC and FcsSC) for short) iff $f(\mu)$ is $r$-fssc (resp. $r$-fssc, $r$-fsso and $r$-fsso) set of $Y$ for each $r$-fsso set (resp. $\tau(\mu) \geq r, r$-fssc and $\tau(\overline{1}-\mu) \geq r$ ), set $\mu \in I^{X} r \in I_{0}$.

(3) fuzzy contra S-homeomorphism (FcS-h, for short) iff $f$ is bijective and both of $f$ and $f^{-1}$ are fuzzy contra S-irresolute continuous.

Theorem 2.2. Let $f:\left(X, \tau_{1}\right) \rightarrow\left(Y, \tau_{2}\right)$ be a mapping. Then the below representaion are equivalent.

(1) $f$ is FCS-ICts.

(2) $\forall r$-fssc $\mu \in I^{Y}, f^{-1}(\mu)$ is $r$-fsso.

(3) $f\left(\operatorname{SSI}_{\tau_{1}}(\lambda, r)\right) \geq \operatorname{SSC}_{\tau_{2}}(f(\lambda), r), \forall \lambda \in I^{X} \& r \in I_{0}$.

(4) $\operatorname{SSI}_{\tau_{1}}\left(f^{-1}(\mu), r\right) \geq f^{-1}\left(\operatorname{SSC}_{\tau_{2}}(\mu, r)\right), \forall \mu \in I^{Y} \& r \in$ $I_{0}$.

(5) $f^{-1}\left(\operatorname{SSI}_{\tau_{2}}(\mu, r)\right) \geq\left(\operatorname{SSC}_{\tau_{1}}\left(f^{-1}(\mu), r\right), \forall \mu \in I^{Y} \& r \in\right.$ $I_{0}$.
Proof. (1) $\Rightarrow(2)$ : For a $r$-fssc set in $\mu \in Y, f^{-1}(\overline{1}-\mu)=$ $\overline{1}-f^{-1}(\mu)$. By (1) $f^{-1}(\overline{1}-\mu)$ is $r$-fssc in $X$. $\Rightarrow \overline{1}-f^{-1}(\mu)$ is $r$-fssc set in $X$. $\Rightarrow f^{-1}(\mu)$ is $r$-fsso set in $X$. Thus proved (2).

(2) $\Rightarrow(3)$ : Assume $f\left(\operatorname{SSI}_{\tau_{1}}(\lambda, r)\right) \ngtr \operatorname{SSC}_{\tau_{2}}(f(\lambda), r)$ for a $\lambda \in I^{X} \& r \in I_{0}$, then $y \in Y$ and $t \in(0,1) \ni f\left(\operatorname{SSI}_{\tau_{1}}(\lambda, r)\right)(y)<$ $t<\operatorname{SSC}_{\tau_{2}}(f(\lambda), r)(y)$. If $f^{-1}(\{y\})=\phi$, because $f\left(\operatorname{SSI}_{\tau_{1}}(\lambda, r)\right)$ $(y)=0$. it is a contradiction. Also $f^{-1}(\{y\}) \neq \phi \exists x \in$ $f^{-1}(\{y\}) \ni$

$$
\begin{aligned}
f\left(\operatorname{SSI}_{\tau_{1}}(\lambda, r)\right)(y) & \leq\left(\operatorname{SSI}_{\tau_{1}}(\lambda, r)(x)\right. \\
& <t \\
& <\operatorname{SSC}_{\tau_{2}}(f(\lambda), r)(f(x))
\end{aligned}
$$

Since $\operatorname{SSC}_{\tau_{2}}(f(\lambda), r)(f(x))>t, \exists r$-fssc $\mu \in I^{Y}$ with $f(\lambda) \geq$ $\mu \ni$

$$
\operatorname{SSC}_{\tau_{2}}(f(\lambda), r)(f(x))>\mu(f(x))>t .
$$

However, $f(\lambda) \geq \mu \Rightarrow \lambda \geq f^{-1}(\mu)$. From (2), $f^{-1}(\mu)$ is $r$ fsso. Thus

$\operatorname{SSI}_{\tau_{1}}(\lambda, r)(x) \geq f^{-1}(\mu)(x)=\mu(f(x))>t$. It contradicts equation 2.1. Hence $f\left(\operatorname{SSI}_{\tau_{1}}(\lambda, r)\right) \geq \operatorname{SSC}_{\tau_{2}}(f(\lambda), r)$.

(3) $\Rightarrow(4): \forall \mu \in I^{Y}, r \in I_{0}$, put $\lambda=f^{-1}(\mu)$.

From (3), we have

$$
\begin{aligned}
f\left(\operatorname{SSI}_{\tau_{1}}\left(f^{-1}(\mu), r\right)\right) & \geq \operatorname{SSC}_{\tau_{2}}\left(f\left(f^{-1}(\mu)\right), r\right) \\
& \geq \operatorname{SSC}_{\tau_{2}}(\mu, r) . \\
\Rightarrow \operatorname{SSI}_{\tau_{1}}\left(f^{-1}(\mu), r\right) & \geq f^{-1}\left(f\left(\operatorname{SSI}_{\tau_{1}}\left(f^{-1}(\mu), r\right)\right)\right) \\
& \geq f^{-1}\left(\operatorname{SSC}_{\tau_{2}}(\mu, r)\right) .
\end{aligned}
$$

Thus $\operatorname{SSI}_{\tau_{1}}\left(f^{-1}(\mu), r\right) \geq f^{-1}\left(\operatorname{SSC}_{\tau_{2}}(\mu, r)\right)$. Thus (4) is proved. (4) $\Rightarrow$ (5): It is evident from Theorem 2.15 in [10]. $S I_{\tau_{1}}\left(f^{-1}(\mu)\right.$, $r) \geq f^{-1}\left(\operatorname{SSC}_{\tau_{2}}(\mu, r)\right)$ We know that by Theorem 2.15 in [10],

$$
\operatorname{SSI}_{\tau}(\lambda, r)=\overline{1}-\operatorname{SSC}_{\tau}(\overline{1}-\lambda, r) .
$$

Hence, we have $\overline{1}-\operatorname{SSC}_{\tau_{1}}\left(\overline{1}-f^{-1}(\mu), r\right) \geq f^{-1}\left(\overline{1}-\operatorname{SSI}_{\tau_{2}}(\overline{1}-\right.$ $\mu, r)) \overline{1}-S S C_{\tau_{1}}\left(\overline{1}-f^{-1}(\mu), r\right) \geq \overline{1}-f^{-1}\left(S S I_{\tau_{2}}(\overline{1}-\mu, r)\right)$ $-\operatorname{SSC}_{\tau_{1}}\left(f^{-1}(\overline{1}-\mu), r\right) \geq-f^{-1}\left(\operatorname{SSI}_{\tau_{2}}(\overline{1}-\mu, r)\right) \quad S S C_{\tau_{1}}$ $\left(f^{-1}(\overline{1}-\mu), r\right) \leq f^{-1}\left(\operatorname{SSI}_{\tau_{2}}(\overline{1}-\mu, r)\right) f^{-1}\left(\operatorname{SSI}_{\tau_{2}}(\overline{1}-\mu, r)\right) \geq$ $S S C_{\tau_{1}}\left(f^{-1}(\overline{1}-\mu), r\right)$. Thus (5) is proved.

(5) $\Rightarrow(1)$ : Let $\mu$ be $r$-fsso set of $Y$. From Theorem ??(3), $\mu=\operatorname{SSI}_{\tau_{2}}(\mu, r)$. By $(5), f^{-1}\left(\operatorname{SSI}_{\tau_{2}}(\mu, r)\right) \geq \operatorname{SSC}_{\tau_{1}}\left(f^{-1}(\mu), r\right)$ $\Rightarrow f^{-1}(\mu) \geq \operatorname{SSC}_{\tau_{1}}\left(f^{-1}(\mu), r\right)$ Also, by Theorem 2.15 in [10], $f^{-1}(\mu) \leq \operatorname{SSC}_{\tau_{1}}\left(f^{-1}(\mu), r\right)$ Thus $f^{-1}(\mu)=\operatorname{SSC}_{\tau_{1}}\left(f^{-1}(\mu)\right.$, $r)$. (i.e) $f^{-1}(\mu)$ is $r$-fssc set in $X$. $\Rightarrow f$ is FcS-ICts.

Thus proved (1).

Theorem 2.3. For a $f:\left(X, \tau_{1}\right) \rightarrow\left(Y, \tau_{2}\right)$, the below representation are equivalent.

(1) A map $f$ is FcsSCts.

(2) For each $r$-fc set $\mu \in I^{Y}, f^{-1}(\mu)$ is $r$-fsso in $X$.

(3) $f\left(\operatorname{SSI}_{\tau_{1}}(\lambda, r)\right) \geq C_{\tau_{2}}(f(\lambda), r), \forall \lambda \in I^{X}$ and $r \in I_{0}$. 
(4) $\operatorname{SSI}_{\tau_{1}}\left(f^{-1}(\mu), r\right) \geq f^{-1}\left(C_{\tau_{2}}(\mu, r)\right), \forall \mu \in I^{Y}$ and $r \in$ $I_{0}$.

Proof. $(1) \Leftrightarrow(2)$ : It is evident from Definition of $r$ - fssc and $f^{-1}(\overline{1}-\mu)=\overline{1}-f^{-1}(\mu)$. Let $\mu$ be $r$ - F closed in $Y$. Then $\overline{1}-\mu$ is $r$ - F open in $Y$. By (1), $f^{-1}(\overline{1}-\mu)$ is $r$-fssc set in $X . \Rightarrow f^{-1}(\overline{1}-\mu)=\overline{1}-f^{-1}(\mu)$ is $r$-fssc in $X . \Rightarrow f^{-1}(\mu)$ is $r$-fsso in $X$. Thus proved (2).

(2) $\Rightarrow(3)$ : Suppose $\exists \lambda \in I^{X}$ and $r \in I_{0} \ni$

$$
f\left(\operatorname{SSI}_{\tau_{1}}(\lambda, r)\right) \ngtr C_{\tau_{2}}(f(\lambda), r) .
$$

There exists $y \in Y$ and $t \in I_{0} \ni f\left(\operatorname{SSI}_{\tau_{1}}(\lambda, r)\right)(y)<t<$ $C_{\tau_{2}}(f(\lambda), r)(y)$. If $f^{-1}(\{y\})=\phi$, it is a contradiction, since

$$
f\left(\operatorname{SSI}_{\tau_{1}}(\lambda, r)\right)(y)=0 .
$$

If $f^{-1}(\{y\}) \neq \phi, \exists x \in f^{-1}(\{y\}) \ni$

$$
\begin{aligned}
f\left(\operatorname{SSI}_{\tau_{1}}(\lambda, r)\right)(y) & \leq \operatorname{SSI}_{\tau_{1}}(\lambda, r)(x) \\
& <t \\
& <C_{\tau_{2}}(f(\lambda), r)(f(x)) .
\end{aligned}
$$

Since $C_{\tau_{2}}(f(\lambda), r)(f(x))>t, \exists r$ - F closed set $\mu \in I^{Y}$ with $f(\lambda) \geq \mu \ni$

$$
C_{\tau_{2}}(f(\lambda), r)(f(x)) \geq \mu(f(x))>t .
$$

However,

$$
f(\lambda) \geq \mu \Rightarrow \lambda \geq f^{-1}(\mu) .
$$

From (2), $f^{-1}(\mu)$ is $r$-fsso. Thus

$$
\operatorname{SSI}_{\tau_{1}}(\lambda, r)(x) \geq f^{-1}(\mu)(x)=\mu(f(x))>t .
$$

It is a contradiction for (2.2). Hence, $f\left(\operatorname{SSI}_{\tau_{1}}(\lambda, r)\right)>C_{\tau_{2}}(f(\lambda)$, $r)$. Hence (3) is proved.

(3) $\Rightarrow(4): \forall \mu \in I^{Y}, r \in I_{0}$, put $\lambda=f^{-1}(\mu)$. From (3), we have

$$
f\left(\operatorname{SSI}_{\tau_{1}}(\lambda, r)\right) \geq C_{\tau_{2}}(f(\lambda), r)
$$

(i.e)

$$
\begin{aligned}
f\left(\operatorname{SSI}_{\tau_{1}}\left(f^{-1}(\mu), r\right)\right) & \geq C_{\tau_{2}}\left(f\left(f^{-1}(\mu)\right), r\right) \\
& \geq C_{\tau_{2}}(\mu, r)
\end{aligned}
$$

It implies

$$
\begin{aligned}
\operatorname{SSI}_{\tau_{1}}\left(f^{-1}(\mu), r\right) & \geq f^{-1}\left(f\left(\operatorname{SSI}_{\tau_{1}}\left(f^{-1}(\mu), r\right)\right)\right) \\
& \geq f^{-1}\left(C_{\tau_{2}}(\mu, r)\right)
\end{aligned}
$$

Thus proved (4).

(4) $\Rightarrow$ (2): Let $\mu$ be $r$-fc in $Y$. Then by (4), $\operatorname{SSI}_{\tau_{1}}\left(f^{-1}(\mu), r\right)$ $\geq f^{-1}(\mu)$. Also, by Theorem 2.15 in [10](5), $f^{-1}(\mu) \geq$ $\operatorname{SSI}_{\tau_{1}}\left(f^{-1}(\mu), r\right)$.

Thus $f^{-1}(\mu)=\operatorname{SSI}_{\tau_{1}}\left(f^{-1}(\mu), r\right)$. (i.e) $f^{-1}(\mu)$ is $r$-fsso. This proves (2).
Theorem 2.4. For a bijective $f:(X, \tau) \rightarrow(Y, \eta)$ the below representation are equivalent.

(1) $f$ is FcS-ICts.

(2) $\operatorname{SSI}_{\eta}(f(\lambda), r) \geq f\left(\operatorname{SSC}_{\tau}(\lambda, r)\right), \forall \lambda \in I^{X} \& r \in I_{0}$.

Proof. (1) $\Leftrightarrow(2)$ : Let $f$ be a FcS-ICts mapping and $\lambda \in I^{X}$ and $r \in I_{0}$. Then $f^{-1}\left(\operatorname{SSI}_{\eta}(f(\lambda), r)\right)$ is $r$-fssc set in $X$. Since $f$ is $1-1$, using Theorem 2.2 we have,

$$
f^{-1}\left(\operatorname{SSI}_{\eta}(\mu, r)\right) \geq \operatorname{SSC}_{\tau}\left(f^{-1}(\mu), r\right) .
$$

Let $\mu=f(\lambda)$. Then, $f^{-1}\left(\operatorname{SSI}_{\eta}(f(\lambda), r)\right) \geq \operatorname{SSC}_{\tau}\left(f^{-1}(f(\lambda))\right.$, $r)=\operatorname{SSC}_{\tau}(\lambda, r)$. Again since $f$ is onto, we have $\operatorname{SSI}_{\eta}(f(\lambda), r)$ $=f f^{-1}\left(\operatorname{SSI}_{\eta}(f(\lambda), r) \geq f\left(\operatorname{SSC}_{\tau}(\lambda, r)\right.\right.$. Thus (2) is proved.

(2) $\Rightarrow(1)$ : Let $\mu$ be $r$-fsso set of $Y$. Then by Theorem 2.15 in [10] (3), $\mu=\operatorname{SSI}_{\eta}(\mu, r)$. By (2),

$$
f\left(\operatorname{SSC}_{\tau}\left(f^{-1}(\mu), r\right) \leq \operatorname{SSI}_{\eta}\left(f\left(f^{-1}(\mu), r\right)\right)=\operatorname{SSI}_{\eta}(\mu, r)=\mu\right.
$$

and $\operatorname{SSC}_{\tau}\left(f^{-1}(\mu), r\right)=f^{-1} f\left(\operatorname{SSC}_{\tau}\left(f^{-1}(\mu), r\right)\right) \leq f^{-1}(\mu)$. Also, by Theorem 2.15 in [10](5), $\operatorname{SSC}_{\tau}\left(f^{-1}(\mu), r\right) \geq f^{-1}(\mu)$. Thus, $f^{-1}(\mu)=\operatorname{SSC}_{\tau}\left(f^{-1}(\mu), r\right)$. (i.e) $f^{-1}(\mu)$ is $r$-fssc. Thus (1).

Theorem 2.5. Let $(X, \tau)$ and $(Y, \eta)$ be fts's and let $f: X \rightarrow Y$ be a mapping. Then the below representation are equivalent.

(1) $f$ is called FcS-IO.

(2) $f\left(\operatorname{SSI}_{\tau}(\lambda, r)\right) \leq \operatorname{SSC}_{\eta}(f(\lambda), r), \forall \lambda \in I^{X}$ and $r \in I_{0}$,

(3) $\operatorname{SSI}_{\tau}\left(f^{-1}(\mu), r\right) \leq f^{-1}\left(\operatorname{SSC}_{\eta}(\mu, r)\right), \forall \mu \in I^{Y}$ and $r \in I_{0}$,

(4) For any $\mu \in I^{Y}$ and any $r$-fssc with $f^{-1}(\mu) \leq \lambda, \exists a$ $r$-fsso $\rho \in I^{Y}$ with $\mu \leq \rho \ni f^{-1}(\rho) \leq \lambda$.

Proof. (1) $\Rightarrow(2)$ : For each $\lambda \in I^{X}$, since, $\operatorname{SSI}_{\tau}(\lambda, r) \leq \lambda$ from Theorem ??(5), we have

$$
f\left(\operatorname{SSI}_{\tau}(\lambda, r)\right) \leq f(\lambda) .
$$

From (1), $f\left(\operatorname{SSI}_{\tau}(\lambda, r)\right)$ is $r$-fssc. Hence

$$
f\left(\operatorname{SSI}_{\tau}(\lambda, r)\right) \leq \operatorname{SSC}_{\eta}(f(\lambda), r) .
$$

Thus proved (2).

(2) $\Rightarrow(3)$ : For all $\mu \in I^{Y}, r \in I_{0}$ put $\lambda=f^{-1}(\mu)$ from (2). Then, $f\left(\operatorname{SSI}_{\tau}\left(f^{-1}(\mu), r\right)\right) \leq \operatorname{SSC}_{\eta}\left(f\left(f^{-1}(\mu)\right), r\right) \leq$ $\operatorname{SSC}_{\eta}(\mu, r)$. It implies

$$
\operatorname{SSI}_{\tau}\left(f^{-1}(\mu), r\right) \leq f^{-1}\left(\operatorname{SSC}_{\eta}(\mu, r)\right) .
$$

(3) $\Rightarrow$ (4): Let $\lambda$ be $r$-fssc set of $X \ni f^{-1}(\mu) \leq \lambda$. Since $\overline{1}-\lambda \leq f^{-1}(\overline{1}-\mu)$ and $\operatorname{SSI}_{\tau}(\overline{1}-\lambda, r)=\overline{1}-\lambda . \operatorname{SSI}_{\tau}(\overline{1}-$ $\lambda, r)=\overline{1}-\lambda \leq \operatorname{SSI}_{\tau}\left(f^{-1}(\overline{1}-\mu, r)\right.$. From (3), $\overline{1}-\lambda \leq$ $\operatorname{SSI}_{\tau}\left(f^{-1}(\overline{1}-\mu, r) \leq f^{-1}\left(\operatorname{SSC}_{\eta}(\overline{1}-\mu, r)\right)\right.$. It implies $\lambda \geq$ $\overline{1}-f^{-1}\left(\operatorname{SSC}_{\eta}(\overline{1}-\mu, r)\right)=f^{-1}\left(\overline{1}-\operatorname{SSC}_{\eta}(\overline{1}-\mu, r)\right)($ i.e) $\lambda \geq$ 
$f^{-1}\left(\operatorname{SSI}_{\eta}(\mu, r)\right)$ Hence $\exists$ a $r$-fsso $\operatorname{SSI}_{\eta}(\mu, r) \in I^{Y}$ with $\mu \leq$ $\operatorname{SSI}_{\eta}(\mu, r) \ni$

$$
f^{-1}\left(\operatorname{SSI}_{\eta}(\mu, r)\right) \leq \lambda
$$

Thus (4) is proved.

$(4) \Rightarrow(1)$ : Let $\omega$ be $r$-fsso set of $X$. Now, we have to prove that $f(\omega)$ is $r$-fssc set of $Y$. Put $\mu=\overline{1}-f(\omega)$ and $\lambda=\overline{1}-\omega$ $\ni \lambda$ is $r$-fssc. We obtain $f^{-1}(\mu)=f^{-1}(\overline{1}-f(\omega))=\overline{1}-$ $f^{-1}(f(\omega)) \leq \overline{1}-\omega=\lambda \Rightarrow f^{-1}(\mu) \leq \lambda$. From (4), $\exists$ a $r$ fsso set $\rho$ with $\mu \leq \rho \ni$

$$
f^{-1}(\rho) \leq \lambda=\overline{1}-\omega .
$$

It implies $\omega \leq \overline{1}-f^{-1}(\rho)=f^{-1}(\overline{1}-\rho)$. Thus,

$$
f(\omega) \leq f\left(f^{-1}(\overline{1}-\rho)\right) \leq \overline{1}-\rho
$$

Also, since $\mu \leq \rho$,

$$
f(\omega)=\overline{1}-\mu \geq \overline{1}-\rho
$$

Hence from equations (2.5) and (2.6), we get, $f(\omega)=\overline{1}-\rho$. (i.e) $f(\omega)$ is $r$-fssc. Thus (1) is proved.

Theorem 2.6. Let $(X, \tau)$ and $(Y, \eta)$ be fts's and $f: X \rightarrow Y$ be a mapping. The below representation are equivalent.

(1) $f$ is called F c S-I closed.

(2) $f\left(\operatorname{SSC}_{\tau}(\lambda, r)\right) \geq \operatorname{SSI}_{\eta}(f(\lambda), r), \forall \lambda \in I^{X}$ and $r \in I_{0}$.

(3) $\operatorname{SSC}_{\tau}\left(f^{-1}(\mu), r\right) \geq f^{-1}\left(\operatorname{SSI}_{\eta}(\mu, r)\right), \forall \mu \in I^{Y}$ and $r \in I_{0}$.

(4) For any $\mu \in I^{Y}$ and $r$-fsso $\lambda \in I^{X}$ with $f^{-1}(\mu) \leq \lambda, \exists$ a $r$-fssc, $\rho \in I^{Y}$ with $\mu \geq \rho \ni f^{-1}(\rho) \geq \lambda$.

Proof. (1) $\Rightarrow(2)$ : Let $f$ be F c S-I closed. For each $\lambda \in I^{X}$ and $r \in I_{0}$, since $\operatorname{SSC}_{\tau}(\lambda, r) \geq \lambda$ from Theorem 2.15 in [10](5), we have

$$
f\left(\operatorname{SSC}_{\tau}(\lambda, r)\right) \geq f(\lambda) .
$$

From (1), $f\left(\operatorname{SSC}_{\tau}(\lambda, r)\right)$ is $r$-fsso. Hence

$$
f\left(\operatorname{SSC}_{\tau}(\lambda, r)\right) \geq \operatorname{SSI}_{\eta}(f(\lambda), r) .
$$

Thus (2) is proved.

(2) $\Rightarrow(3)$ : For all $\mu \in I^{Y}, r \in I_{0}$ put $\lambda=f^{-1}(\mu)$ from (2). Then,

$$
f\left(\operatorname{SSC}_{\tau}\left(f^{-1}(\mu), r\right)\right) \geq \operatorname{SSI}_{\eta}\left(f\left(f^{-1}(\mu)\right), r\right) \geq \operatorname{SSI}_{\eta}(\mu, r) .
$$

It implies $\operatorname{SSC}_{\tau}\left(f^{-1}(\mu), r\right) \geq f^{-1}\left(\operatorname{SSI}_{\eta}(\mu, r)\right)$. Thus proved (3) .

(3) $\Rightarrow(4)$ : Let $\lambda$ be $r$-fsso set of $X \ni f^{-1}(\mu) \geq \lambda$. Since $\overline{1}-\lambda \geq f^{-1}(\overline{1}-\mu)$ and $\operatorname{SSC}_{\tau}(\overline{1}-\lambda, r)=\overline{1}-\lambda \operatorname{SSC}_{\tau}(\overline{1}-$ $\lambda, r)=\overline{1}-\lambda \geq S S C_{\tau}\left(f^{-1}(\overline{1}-\mu, r)\right.$. From (3), $\overline{1}-\lambda \geq$ $\operatorname{SSC}_{\tau}\left(f^{-1}(\overline{1}-\mu), r\right) \geq f^{-1}\left(\operatorname{SSI}_{\eta}(\overline{1}-\mu, r)\right)$ It implies $\lambda \leq$ $\overline{1}-f^{-1}\left(\operatorname{SSI}_{\eta}(\overline{1}-\mu, r)\right)=f^{-1}\left(\overline{1}-\operatorname{SSI}_{\eta}(\overline{1}-\mu, r)\right) \quad \lambda \leq$ $f^{-1}\left(\operatorname{SSC}_{\eta}(\mu, r)\right)$ Hence $\exists$ a $r$-fssc set $\operatorname{SSC}_{\eta}(\mu, r) \in I^{Y}$ with $\mu \geq \operatorname{SSC}_{\eta}(\mu, r) \ni f^{-1}\left(\operatorname{SSC}_{\eta}(\mu, r)\right) \geq \lambda$.
(4) $\Rightarrow(1)$ : Let $\omega$ be $r$-fssc set of $X$. Put $\mu=\overline{1}-f(\omega)$ and $\lambda=\overline{1}-\omega \ni \lambda$ is $r$-fsso. We obtain $f^{-1}(\mu)=f^{-1}(\overline{1}-$ $f(\omega))=\overline{1}-f^{-1}(f(\omega)) \leq \overline{1}-\omega=\lambda$. From (4), $\exists$ a $r$-fssc set $\rho \in I^{Y}$ with $\mu \geq \rho \ni$

$$
f^{-1}(\rho) \geq \lambda=\overline{1}-\omega .
$$

It implies $\omega \geq \overline{1}-f^{-1}(\rho)=f^{-1}(\overline{1}-\rho)$. Thus,

$$
f(\omega) \geq f\left(f^{-1}(\overline{1}-\rho)\right)=\overline{1}-\rho .
$$

Also, since $\mu \leq \rho$,

$$
f(\omega)=\overline{1}-\mu \leq \overline{1}-\rho
$$

Hence from (2.7) and (2.8), we have $f(\omega)=\overline{1}-\rho$. (i.e) $f(\omega)$ is $r$-fsso. Thus (1).

Theorem 2.7. Let $(X, \tau)$ and $(Y, \eta)$ befts's and let $f: X \rightarrow Y$ be a bijective mapping. Then the below representation hold:

(1) $f$ is a F c S-I closed iff $f^{-1}\left(\operatorname{SSI}_{\eta}(\mu, r) \leq \operatorname{SSC}_{\tau}\left(f^{-1}(\mu)\right.\right.$, $r), \forall \mu \in I^{Y}$ and $r \in I_{0}$,

(2) $f$ is F c S-I closed iff $f$ if F c S-I open.

Proof. (1) $\Rightarrow$ : Let $f$ be F c S-I closed. From Theorem 2.6(2), $\forall \lambda \in I^{X}$ and $r \in I_{0}$,

$$
f\left(\operatorname{SSC}_{\tau}(\lambda, r)\right) \geq \operatorname{SSI}_{\eta}(f(\lambda), r) .
$$

For all $\mu \in I^{Y}, r \in I_{0}$. Put $\lambda=f^{-1}(\mu)$, since $f$ is onto, $f\left(f^{-1}(\mu)\right)=\mu$. Thus,

$$
f\left(\operatorname{SSC}_{\tau}\left(f^{-1}(\mu), r\right)\right) \geq \operatorname{SSI}_{\tau}\left(f\left(f^{-1}(\mu)\right), r\right)=\operatorname{SSI}_{\eta}(\mu, r) .
$$

It implies

$$
\begin{aligned}
\operatorname{SSC}_{\tau}\left(f^{-1}(\mu), r\right) & =f^{-1}\left(f\left(\operatorname{SSC}_{\tau}\left(f^{-1}(\mu), r\right)\right)\right) \\
& \geq f^{-1}\left(\operatorname{SSI}_{\eta}(\mu, r)\right) .
\end{aligned}
$$

$(\Leftarrow)$ Put $\mu=f(\lambda)$. Since $f$ is injective, $f^{-1}\left(\operatorname{SSI}_{\eta}(f(\lambda), r)\right) \leq$ $\operatorname{SSC}_{\tau}\left(f^{-1}(f(\lambda)), r\right)=\operatorname{SSC}_{\tau}(\lambda, r)$. Since $f$ is onto,

$$
\operatorname{SSI}_{\eta}(f(\lambda), r) \leq f\left(\operatorname{SSC}_{\tau}(\lambda, r)\right) .
$$

$\Rightarrow f$ is F c S-I closed. (2) It is evident from : $f^{-1}\left(\operatorname{SSI}_{\eta}(\mu, r)\right)$ $\leq \operatorname{SSC}_{\tau}\left(f^{-1}(\mu), r\right) \Leftrightarrow f^{-1}\left(\overline{1}-\operatorname{SSC}_{\eta}(\overline{1}-\mu, r)\right) \leq \overline{1}-$ $\operatorname{SSI}_{\tau}\left(\overline{1}-f^{-1}(\mu), r\right) \Leftrightarrow \overline{1}-f^{-1}\left(\operatorname{SSC}_{\eta}(\overline{1}-\mu, r)\right) \leq \overline{1}-$ $\operatorname{SSI}_{\tau}\left(f^{-1}(\overline{1}-\mu), r\right) \Leftrightarrow f^{-1}\left(\operatorname{SSC}_{\eta}(\overline{1}-\mu, r)\right) \geq \operatorname{SSI}_{\tau}\left(f^{-1}(\overline{1}-\right.$ $\mu), r) \Leftrightarrow$ Hence $f$ is F c S-I open.

Theorem 2.8. Let $f:(X, \tau) \rightarrow(Y, \eta)$ be a bijective mapping from an fts $(X, \tau)$ into an fts $(Y, \eta)$. Then the below representation are equivalent.

(1) $f$ is F c S-I homeomorphism.

(2) $f$ is F c S-I Cts and F c S-I closed.

(3) $f$ is F c S-I Cts and F c S-I open. 
(4) $f\left(\operatorname{SSI}_{\tau}(\lambda, r)\right)=\operatorname{SSC}_{\eta}(f(\lambda), r), \forall \lambda \in I^{X}$ and $r \in I_{0}$.

(5) $f\left(\operatorname{SSC}_{\tau}(\lambda, r)\right)=\operatorname{SSI}_{\eta}(f(\lambda), r), \forall \lambda \in I^{X}$ and $r \in I_{0}$.

(6) $\operatorname{SSI}_{\tau}\left(f^{-1}(\mu), r\right)=f^{-1}\left(\operatorname{SSC}_{\eta}(\mu, r)\right), \forall \mu \in I^{Y}$ and $r \in I_{0}$.

(7) $\operatorname{SSC}_{\tau}\left(f^{-1}(\mu), r\right)=f^{-1}\left(\operatorname{SSI}_{\eta}(\mu, r)\right), \forall \mu \in I^{Y}$ and $r \in I_{0}$.

Proof. (1) $\Rightarrow(2)$ : Since $f$ is c S-I homeomorphism, $f$ and $f^{-1}$ are c S-I Cts. Let $\mu$ be any $r$-fsso set in $Y$. As $f$ is c S-I Cts, $f^{-1}(\mu)$ is $r$-fssc set of $X$. Also $f^{-1}$ is c S-I Cts implies that $f\left(f^{-1}(\mu)\right)=\mu$ is $r$-fsso set in $Y$. This implies that $f$ is c S-I closed map.

(2) $\Rightarrow(1)$ : Let $\mu$ be any $r$-fsso set of $Y$. Since $f$ is F c S-I Cts, $f^{-1}(\mu)$ is $r$-fssc set of $Y$. Since $f$ is F c S-I closed map, $f\left(f^{-1}(\mu)\right)=\mu$ is $r$-fsso in $X$. Thus $f^{-1}$ is F c S-I Cts.

$(2) \Leftrightarrow(3)$ : It is clear from Theorem 2.7(2).

(2) $\Leftrightarrow(5)$ : For each $\lambda \in I^{X}$ and $r \in I_{0}$, since $f$ is c S-I closed, using Theorem2.6(2), we have,

$$
f\left(\operatorname{SSC}_{\tau}(\lambda, r)\right) \geq \operatorname{SSI}_{\eta}(f(\lambda), r) .
$$

Also $f$ is F c S-I Cts, so by Theorem2.4,

$$
\operatorname{SSI}_{\eta}(f(\lambda), r) \geq f\left(\operatorname{SSC}_{\tau}(\lambda, r)\right),
$$

$\forall \lambda \in I^{X}$ and $r \in I_{0}$. Hence, $f\left(\operatorname{SSC}_{\tau}(\lambda, r)\right)=\operatorname{SSI}_{\eta}(f(\lambda), r)$.

(3) $\Leftrightarrow(4)$ : For each $\lambda \in I^{X}$ and $r \in I_{0}$, since $f$ is c S-I open, using Theorem 2.5(2), we have,

$$
f\left(\operatorname{SSI}_{\tau}(\lambda, r)\right) \leq \operatorname{SSC}_{\eta}(f(\lambda), r) .
$$

Also $f$ is F c S-I Cts, so by Theorem 2.2, $f\left(\operatorname{SSI}_{\tau}(\lambda, r)\right) \geq$ $\operatorname{SSC}_{\eta}(f(\lambda), r), \forall \lambda \in I^{X}$ and $r \in I_{0}$. Hence, $f\left(\operatorname{SSI}_{\tau}(\lambda, r)\right)=$ $\operatorname{SSC}_{\eta}(f(\lambda), r)$.

(2) $\Leftrightarrow(7)$ : Let $f$ be F c S-I Cts and $\lambda \in I^{X}, r \in I_{0}$. Then from Theorem 2.4,

$$
\operatorname{SSI}_{\eta}(f(\lambda), r) \geq f\left(\operatorname{SSC}_{\tau}(\lambda, r)\right) .
$$

Since $f$ is c S-I closed, by Theorem 2.6(3),

$$
\operatorname{SSC}_{\tau}\left(f^{-1}(\mu), r\right) \geq f^{-1}\left(\operatorname{SSI}_{\eta}(\mu, r)\right),
$$

$\forall \mu \in I^{Y}$ and $r \in I_{0}$. By putting $\lambda=f^{-1}(\mu), \forall \mu \in I^{Y}$ and $r \in I_{0}$. Thus proves (7).

(3) $\Leftrightarrow(6)$ : Let $f$ be F c S-I Cts and $\lambda \in I^{X}, r \in I_{0}$. Then from Theorem 2.2,

$$
f\left(\operatorname{SSI}_{\tau}(\lambda, r)\right) \geq \operatorname{SSC}_{\eta}(f(\lambda), r) .
$$

Since $f$ is c S-I open, by Theorem 2.5 ,

$$
\operatorname{SSI}_{\tau}\left(f^{-1}(\mu), r\right) \leq f^{-1}\left(\operatorname{SSC}_{\eta}(\mu, r)\right),
$$

$\forall \mu \in I^{Y}$ and $r \in I_{0}$. By putting $\lambda=f^{-1}(\mu), \forall \mu \in I^{Y}$ and $r \in I_{0}$. Thus proves (6).
Remark 2.9. For a mapping $f: X \rightarrow Y$, the below representation are valid:

(1) $f$ is FcS-ICts $\Rightarrow f$ is FcsSCts.

(2) $f$ is FcS-ICts $\Rightarrow f$ is FcCts.

(3) $f$ is FcS-IO (resp. FcS-IC) $\Rightarrow f$ is FcsSO (resp. Fc$s S C)$.

(4) $f$ is $F c S-I O$ (resp. FcS-IC) $\Rightarrow f$ is $F c O$ (resp. FcC).

But the converses need not be true as shown by the following examples.

\section{Example}

Example 3.1. Consider the fts's $(X, \tau) \&(Y, \eta)$ with $X=Y=$ $\{x, y, z\}$ and

$\tau(\lambda)=\left\{\begin{array}{l}1, \text { if } \lambda=\overline{0} \text { or } \overline{1} \\ \frac{1}{2}, \text { if } \lambda=v_{1} \\ 0, \text { otherwise }\end{array} \quad \eta(v)=\left\{\begin{array}{l}1, \text { if } \lambda=\overline{0} \text { or } \overline{1} \\ \frac{1}{2}, \text { if } v=v_{2} \\ 0, \text { otherwise }\end{array}\right.\right.$

where $v_{1}(x)=0.3, v_{1}(y)=0.3, v_{1}(z)=0.5 ; v_{2}(x)=0.7, v_{2}(y)=$ $0.7, v_{2}(z)=0.5 ; \quad v_{3}(x)=0.8, \quad v_{3}(y)=0.8, v_{3}(z)=0.5$.

Then the identity function $f: X \rightarrow Y$ is

(i) FcsSCts but not FcS-ICts because $v_{3}$ is $\frac{1}{2}$-fsso in $(Y, \eta)$ but $f^{-1}\left(v_{3}\right)=v_{3}$ is not $\frac{1}{2}$-fssc in $(X, \tau)$.

(ii) FcCts but not FcS-ICts because $v_{3}$ is $\frac{1}{2}-f$ sso in $(Y, \eta)$ but $f^{-1}\left(v_{3}\right)=v_{4}$ is not $\frac{1}{2}$-fssc in $(X, \tau)$.

Example 3.2. Consider the fts's $(X, \tau) \&(Y, \eta)$ with $X=Y=$ $\{x, y, z\}$ and

$\tau(v)=\left\{\begin{array}{l}1, \text { if } v=\overline{0} \text { or } \overline{1} \\ \frac{1}{2}, \text { if } v=v_{1} \\ 0, \text { otherwise }\end{array}\right.$

$\eta(\mu)=\left\{\begin{array}{l}1, \text { if } \mu=\overline{0} \text { or } \overline{1} \\ \frac{1}{2}, \text { if } \mu=v_{2} \\ 0, \text { otherwise }\end{array}\right.$

$v_{1}(x)=0.7, v_{1}(y)=0.7, v_{1}(z)=0.5$;

$v_{2}(x)=0.3, v_{2}(y)=0.3, v_{2}(z)=0.5$;

$v_{3}(x)=0.8, v_{3}(y)=0.8, v_{3}(z)=0.5$.

Then the identity function $f: X \rightarrow Y$ is FcsSO but not FcS-IO because $v_{3}$ is $\frac{1}{2}-f$ sso in $(X, \tau)$ but $f\left(v_{3}\right)=v_{3}$ is not $\frac{1}{2}$-fssc in $(Y, \eta)$.

Theorem 3.3. If $f: X \rightarrow Y$ is FcsSO and $g: Y \rightarrow Z$ is FcS-IC, then $g \circ f: X \rightarrow Z$ is FsSO map.

Proof. Let $\lambda$ be $r$ - fo set in $X$. As $f$ is FcsSO, $f(\lambda)$ is $r$-fssc set of $Y$. Also since $g: Y \rightarrow Z$ is FcS-IC, $g(f(\lambda))$ is $r$-fsso in $Z$. (i.e) $(g \circ f)(\lambda)=g(f(\lambda))$ is $r$-fsso in $Z$. Thus $g \circ f$ is FsSO map. 
Theorem 3.4. If $f: X \rightarrow Y$ is FcS-IO and $g: Y \rightarrow Z$ is FcS-IC, then $g \circ f: X \rightarrow Z$ is FS-IO map.

Proof. Let $\lambda$ be $r$-fsso set in $X$. As $f$ is FcS-IO, $f(\lambda)$ is $r$-fssc set of $Y$. Also since $g: Y \rightarrow Z$ is F c S-I closed, $g(f(\lambda))$ is $r$-fsso in $Z$. (i.e)

$$
(g \circ f)(\lambda)=g(f(\lambda))
$$

is $r$-fsso in $Z$. Thus $g \circ f$ is FS-IO map.

Theorem 3.5. If $f: X \rightarrow Y$ is $F c O$ and $g: Y \rightarrow Z$ is FcsSC, then $g \circ f: X \rightarrow Z$ is FsSO map.

Proof. Let $\mu$ be $r$-fo set in $X$. As $f$ is FcO, $f(\mu)$ is $r$-fc set of $Y$. Also since $g: Y \rightarrow Z$ is FcsSC, $g(f(\mu))$ is $r$-fsso in $Z$. (i.e) $(g \circ f)(\mu)=g(f(\mu))$ is $r$-fsso in $Z$. Thus $g \circ f$ is FsSO map.

Theorem 3.6. If $f: X \rightarrow Y$ is FcC and $g: Y \rightarrow Z$ is FcsSO, then $g \circ f: X \rightarrow Z$ is FsSC map.

Proof. Let $\mu$ be $r$ - fc set in $X$. As $f$ is FcC, $f(\mu)$ is $r$ - fo set of $Y$. Also since $g: Y \rightarrow Z$ is FcsSO, $g(f(\mu))$ is $r$-fssc in $Z$. (i.e) $(g \circ f)(\mu)=g(f(\mu))$ is $r$-fssc in $Z$. Thus $g \circ f$ is FsSC map.

Theorem 3.7. If $f: X \rightarrow Y$ is FcS-ICts and $: Y \rightarrow Z g$ is FSICts, then $g \circ f: X \rightarrow Z$ is FcS-ICts.

Proof. Let $\mu$ be $r$-fsso set in $Z$. As $g$ is FS-ICts, $g^{-1}(\mu)$ is $r$-fsso set of $Y$. Also since $f: Y \rightarrow Z$ is FcS-ICts, $f^{-1}\left(g^{-1}(\mu)\right)$ is $r$-fssc in $X$. (i.e) $(g \circ f)^{-1}(\mu)=f^{-1}\left(g^{-1}(\mu)\right)$ is $r$-fssc set in $X$. Thus $g \circ f$ is FcS-I.

\section{Conclusion}

Šostak's fuzzy topology has been recently of major interest among fuzzy topologies. The concepts of FcS-ICts, FcsSCts,FcS-IO, FcsSO, FcS-IC, FcsSC and FcS-H in fuzzy topological space in Šostak's sense are introduced and studied. Some of their characteristic properties are considered. Also a comparison between these new types of functions are established and counter examples are also given. These results will help to extend the some generalized continuous mappings, compactness and hence it will help to improve smooth topological and bi-topological spaces.

\section{References}

[1] C. L. Chang, Fuzzy topological spaces, J. Math. Anal. Appl., 24 (1968), 182-189.

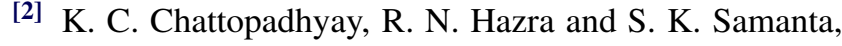
Gradation of openness, Fuzzy Sets and Systems, 49 (2) (1992), 237-242.

${ }^{[3]}$ K. C. Chattopadhyay and S. K. Samanta, Fuzzy topology: fuzzy closure operator, fuzzy compactness and fuzzy connectedness, Fuzzy Sets and Systems, 54 (2) (1993), 207-212.
[4] R. N. Hazra, S. K. Samanta and K. C. Chattopadhyay, Fuzzy topology redefined, Fuzzy Sets and Systems, 4 (1992), 79-82.

[5] U. Höhle, Upper semicontinuous fuzzy sets and applications, J. Math. Anall. Apll., 78 (1980), 659-673.

[6] U. Höhle and A. P. Šostak, A general theory of fuzzy topological spaces, Fuzzy sets and systems, 73 (1995), 131-149.

${ }^{[7]}$ U. Höhle and A. P. Šostak, Axiomatic Foundations of Fixed-Basis fuzzy topology, The Handbooks of Fuzzy sets series, Volume 3, Kluwer Academic Publishers, Dordrecht (Chapter 3)(1999), 123-272.

[8] T. Kubiak, On fuzzy topologies, Ph.D. Thesis, A. Mickiewicz, Poznan, (1985).

[9] T. Kubiak and A. P. Šostak, Lower set-valued fuzzy topologies, Quaestions Math., 20 (3) (1997), 423-429.

[10] Y. C. Kim, A. A. Ramadam and S. E. Abbas, Weaker forms of continuity in Šostak fuzzy topology, Indian J. Pure Appl. Math., 34(2) (2003), 311-333.

[11] A. A. Ramadan, Smooth topological spaces, Fuzzy Sets and Systems 48 (1992), 371-375.

${ }^{[12]}$ S. E. Rodabaugh, Categorical foundations of variable basis topology, in U. Höhle, S. E. Rodabaugh(Eds)), The Handbooks of fuzzy sets series, 3, Fuzzy sets : Logic, topology and Measure Theory, Kluwer Academic Publishers, Dordrecht (1991).

[13] A. P. Šostak, On a fuzzy topological structure, Rend. Circ. Matem. Palermo Ser.II 11 (1985), 89-103.

[14] A. P. Šostak, Two decades of fuzzy topology : Basic ideas, Notion and results, Russian Math. Surveys, 44 (6) (1989), 125-186.

[15] A. P. Šostak, On some modifications of fuzzy topologies, Mathematicki vesnik, 41 (1989), 20-37.

[16] A. P. Šostak, On the neighbourhood structure of fuzzy topologies, Zb. Radova Univ. Nisu, 4 (1999), 7-14.

${ }^{[17]}$ M. S. Ying, A new approach for fuzzy topology (I), Fuzzy sets and systems, 39 (1991), 303-321.

${ }^{[18]}$ L. A. Zadeh, Fuzzy sets, Inform. Control, 8 (1965), 338353. 